\title{
Estimating Invariant Measures and Lyapunov Exponents
}

\author{
Brian R. Hunt ${ }^{1}$ \\ Institute for Physical Science and Technology \\ University of Maryland \\ College Park, MD 20742 \\ bhunt@ipst.umd.edu \\ March 10, 1995
}

\begin{abstract}
This paper describes a method for obtaining rigorous numerical bounds on time averages for a class of one-dimensional expanding maps. The idea is to directly estimate the absolutely continuous invariant measure for these maps, without computing trajectories. The main theoretical result is a bound on the convergence rate of the Frobenius-Perron operator for such maps. The method is applied to estimate the Lyapunov exponents for a planar map of recent interest.
\end{abstract}

\section{Introduction}

When one studies an attractor of a chaotic dynamical system quantitatively, one is often interested in estimating a "time average": the average of a given function of the state of the system over a typical trajectory on, or approaching, the attractor. Of particular interest are Lyapunov exponents, which reflect average rates of linear expansion or contraction near the attractor. In some very special cases, Lyapunov exponents can be determined exactly, but typically they are measured with the aid of a computer. In this paper, we show how a careful numerical study can yield a close rigorous estimate of a Lyapunov exponent or other time average for certain dynamical systems.

\footnotetext{
${ }^{1}$ The author was supported by the National Science Foundation (Divisions of Mathematical and Physical Sciences) and by the U.S. Department of Energy (Offices of Scientific Computing and Energy Research).
} 
One approach to estimating a time average is of course to compute one or several computer trajectories near the attractor and find the average over the computed points. See [6] for a general discussion of the computation of Lyapunov exponents in this manner. This approach is subject to statistical sampling error as well as the error due to numerical computation of trajectories. Here we describe an approach based on directly estimating the invariant measure supported by the attractor. This allows us to obtain rigorous bounds on the the error in the computation.

Our results concern one-dimensional maps $\tau:[0,1] \rightarrow[0,1]$ which are piecewise $C^{2}$. In the case we study, there will be a unique probability measure $\tilde{\mu}$ which is invariant under $\tau$ and is absolutely continuous with respect to Lebesgue measure. The Birkhoff ergodic theorem then implies that for almost every initial condition $x_{0}$, the forward trajectory of $x_{0}$ is asymptotically distributed according to $\mu$. That is, if $\varphi:[0,1] \rightarrow \mathbf{R}$ is continuous, then for almost every $x_{0}$ the time average of $\varphi$ over the trajectory of $x_{0}$ is given by

$$
\lim _{n \rightarrow \infty} \frac{1}{n} \sum_{k=0}^{n-1} \varphi\left(\tau^{k}\left(x_{0}\right)\right)=\int_{M} \varphi(x) d \tilde{\mu}(x) .
$$

Our goal is to approximate $\int \varphi d \tilde{\mu}$ for a given $\tau$ and $\varphi$. Since $\tilde{\mu}$ is absolutely continuous, by the Radon-Nikodym theorem we can write $d \tilde{\mu}(x)=\tilde{f}(x) d x$, where $\tilde{f}$ is a nonnegative $L^{1}$ function with integral one. We call $\tilde{f}$ the invariant density of $\tau$. Since $\tilde{\mu}$ is invariant under $\tau$, it follows that $\tilde{f}$ is a fixed point of the Frobenius-Perron operator $P_{\tau}: L^{1}[0,1] \rightarrow L^{1}[0,1]$, defined by

$$
P_{\tau} f(x)=\sum_{\{y: \tau(y)=x\}} f(y) \gamma(y)
$$

where

$$
\gamma(x)=\left\{\begin{array}{cl}
1 / \tau^{\prime}(x) & \text { if } \tau \text { is } C^{1} \text { at } x \\
0 & \text { otherwise }
\end{array} .\right.
$$

Since the invariant density $\tilde{f}$ is unique, one can hope to approximate $\tilde{f}$ by iterating $P_{\tau}$ a number of times using a uniform initial density $f_{0} \equiv 1$. If one does the iteration on a computer, one may find a density $f$ which is invariant, or very nearly invariant, under the computer implementation of $P_{\tau}$. If so, we would like to be able to show that $f$ is close to $\tilde{f}$.

By examining carefully the computer implementation of $P_{\tau}$, we should be able to show that $f$ is close to $P_{\tau} f$. Assume that we can also show that $P_{\tau}$ is a contraction for some norm $\|\cdot\|$, that is

$$
\left\|P_{\tau} g-P_{\tau} h\right\| \leq \rho\|g-h\|
$$

for some $\rho<1$ and all $g, h$ in some function space containing $f$ and $\tilde{f}$. It then will follow that

$$
\|f-\tilde{f}\| \leq\left\|f-P_{\tau} f\right\|+\left\|P_{\tau} f-\tilde{f}\right\|=\left\|f-P_{\tau} f\right\|+\left\|P_{\tau} f-P_{\tau} \tilde{f}\right\| \leq\left\|f-P_{\tau} f\right\|+\rho\|f-\tilde{f}\|,
$$


whence

$$
\|f-\tilde{f}\| \leq \frac{1}{1-\rho}\left\|f-P_{\tau} f\right\|
$$

Thus our procedure for estimating the average

$$
\int_{0}^{1} \varphi(x) \tilde{f}(x) d x
$$

will be to find an approximate invariant density $f$, to integrate $\varphi f$, and to bound the error using the above bound on the difference between $f$ and $\tilde{f}$. To obtain a bound on the error, it is essential that we know what norm $P_{\tau}$ contracts and that we are able to bound the contraction rate $\rho$ away from 1 .

Prior work on the convergence of $P_{\tau}$ has tended to use a spectral approach to show that $P_{\tau}^{n} f_{0}-\tilde{f}$ decays exponentially as $n \rightarrow \infty$; see e.g. $[4,7,9,16,21]$. More recently Rychlik and Sorets $[17,18]$ proved for certain expanding maps $\tau$ that $P_{\tau}$ is a contraction for a specific norm and showed how to put an upper bound on the contraction rate. The goal in those papers was to prove that the contraction rate is uniform for certain families of maps. In this paper the goal will be to make an explicit bound on the contraction rate for a more specific class of expanding maps. For the sake of our application, the bound on the contraction rate must not be extremely close to one.

Another approach to approximating invariant measures involves partitioning the interval, making a Markov approximation to $P_{\tau}$, and finding the fixed point of the approximation; see e.g. $[5,11]$. This approach can be useful in cases where the map is not uniformly expanding [13] and in cases where data for the system is available but a formula for the map is not known [8]. Of course by using a computer approximation to $P_{\tau}$ in this paper we are also discretizing the interval, but our approach differs in that we are not concerned with the fixed point of the discretized Frobenius-Perron operator and work as much as possible in terms of the exact operator.

The following assumptions will be made throughout this paper.

1. The map $\tau:[0,1] \rightarrow[0,1]$ is piecewise $C^{2}$.

2. If $\tau$ is not $C^{2}$ at $x$, then $\tau(x)$ is either 0 or 1 (that is, all branches of $\tau$ are onto).

3. There are real constants $q, r, s$ such that $1<q \leq\left|\tau^{\prime}\right| \leq r$ and $\left|\tau^{\prime \prime}\right| / \tau^{\prime 2} \leq s$.

This class of functions includes nonlinear perturbations of familiar maps like the tent map and the map $x \mapsto k x(\bmod 1)$ for integers $k>1$. For our computer estimates, we will have to assume further that $\tau$ is $C^{3}$ on each of its branches.

In Section 2 we will describe how to find, in terms of $q, r$, and $s$, a norm and a contraction rate for $P_{\tau}$ which will be valid on the space of functions of bounded variation. Then in Section 
3 we describe how to use this result to obtain rigorous bounds on the error for a computer estimate of the invariant density. Finally, in Section 4 we apply this procedure to a specific example: the quadratic map with a particular parameter value studied previously by Ruelle [15]. In particular, we estimate a time average for this map which can be shown to be a Lyapunov exponent for a two-dimensional map studied in $[2,1]$. In the latter paper it is vital to know rigorously that the Lyapunov exponent is negative.

\section{Contraction rate of the Frobenius-Perron operator}

Let $\tau:[0,1] \rightarrow[0,1]$ be piecewise $C^{2}$ with $1<q \leq\left|\tau^{\prime}\right| \leq r$ and $\left|\tau^{\prime \prime}\right| / \tau^{\prime 2}<s$ for some constants $q, r, s$. Assume that all branches of $\tau$ are onto; in other words, if $\tau$ is not $C^{2}$ at $x$ then $\tau(x)=0$ or $\tau(x)=1$. The Frobenius-Perron operator $P_{\tau}$ is defined on $L^{1}[0,1]$ by

$$
P_{\tau} f(x)=\sum_{\tau(y)=x} \frac{f(y)}{\left|\tau^{\prime}(y)\right|} .
$$

(Notice that the condition that all branches of $\tau$ are onto implies that if $f$ is continuous, so is $P_{\tau} f$.) If $f$ represents an initial probability density of points in $[0,1]$, then $P_{\tau} f$ is the density that results after iterating $\tau$ once.

Let $S \subset L^{1}[0,1]$ be the space of functions of bounded variation on $[0,1]$; every $f \in S$ is differentiable almost everywhere with $f^{\prime} \in L^{1}[0,1]$, and the total variation $\operatorname{var}(f)$ of $f$ is equal to the $L^{1}$ norm of $f^{\prime}$. It can be shown (see the proof of Lemma 1 below) that

$$
\operatorname{var}\left(P_{\tau} f\right) \leq \frac{1}{q} \operatorname{var}(f)+s\|f\|_{1} .
$$

where $\|f\|_{1}$ denotes the $L^{1}$ norm of $f$. In particular, if $f \in S$ then $P_{\tau} f \in S$. Furthermore, it is not hard to show that the integral of $P_{\tau} f$ equals the integral of $f$, so if $S_{1}$ denotes the set of probability densities (nonnegative functions with integral one) in $S$ then $P_{\tau}$ maps $S_{1}$ into itself.

An estimate similar to (2) is used in [10] to prove (for a more general class of maps) the existence of a unique invariant density $\tilde{f} \in S_{1}$ (that is, a unique $\tilde{f} \in S_{1}$ for which $P_{\tau} \tilde{f}=\tilde{f}$ ). Here we will show, for the class of maps described above, that repeated iteration of $P_{\tau}$ on an initial function in $S_{1}$ converges to $\tilde{f}$, and explicitly estimate the rate of convergence with respect to a particular norm. Given $f \in S_{1}$, let $g=f-\tilde{f}$; then $P_{\tau}^{n} g=P_{\tau}^{n} f-\tilde{f}$ for all $n \geq 0$. Let $S_{0}$ be the set of functions in $S$ with integral zero; then $g \in S_{0}$. The following lemma will be used to show that $P_{\tau}^{n} g \rightarrow 0$ as $n \rightarrow \infty$.

Lemma 1 (Vector Inequality Lemma) For all $g \in S_{0}$,

$$
\operatorname{var}\left(P_{\tau} g\right) \leq \frac{1}{q} \operatorname{var}(g)+s\|g\|_{1}
$$




$$
\left\|P_{\tau} g\right\|_{1} \leq \frac{1}{2 r} \operatorname{var}(g)+\frac{r-q}{r}\|g\|_{1} .
$$

We prove Lemma 1 at the end of this section. Our present objective is to determine when (3) and (4) can be used to show that $P_{\tau} g$ is always smaller than $g$ in some norm. Let $M$ be the matrix

$$
M=\left(\begin{array}{cc}
\frac{1}{q} & s \\
\frac{1}{2 r} & \frac{r-q}{r}
\end{array}\right) .
$$

Then coordinate-wise,

$$
\left(\begin{array}{c}
\operatorname{var}(P g) \\
\|P g\|_{1}
\end{array}\right) \leq M\left(\begin{array}{c}
\operatorname{var}(g) \\
\|g\|_{1}
\end{array}\right)
$$

Since $M$ has positive entries (we ignore the trivial case $s=0$ ), the eigenvalues of $M$ are real. Further, by a result of Perron \& Frobenius (see [20]), the greatest eigenvalue $\rho$ of $M$ has a left eigenvector $(\alpha, \beta)$ with positive coordinates. Multiplying the above inequality on the left by $(\alpha, \beta)$ we find that

$$
\alpha \operatorname{var}(P g)+\beta\|P g\|_{1} \leq(\alpha, \beta) M\left(\begin{array}{c}
\operatorname{var}(g) \\
\|g\|_{1}
\end{array}\right)=\rho(\alpha, \beta)\left(\begin{array}{c}
\operatorname{var}(g) \\
\|g\|_{1}
\end{array}\right)=\rho\left(\alpha \operatorname{var}(g)+\beta\|g\|_{1}\right) .
$$

This motivates the definition of the " $(\alpha, \beta)$-norm"

$$
\|g\|_{\alpha, \beta}=\alpha \operatorname{var}(g)+\beta\|g\|_{1} .
$$

We then know that $P$ is a contraction in this norm as long as $\rho<1$.

Theorem 2 If $1+s / 2<q$, then the greatest eigenvalue $\rho$ of the matrix $M$ above is less than one, and for all $g \in S_{0}$,

$$
\|P g\|_{\alpha, \beta} \leq \rho\|g\|_{\alpha, \beta}
$$

where $(\alpha, \beta)$ is the left eigenvector of $M$ corresponding to $\rho$.

Proof We have already proved (5), so we need only show that $\rho<1$. Since the trace of $M$ is less than 2, the characteristic polynomial of $M$ takes on its minimum at a value less than 1 , and thus both eigenvalues will be less than 1 provided the characteristic polynomial of $M$ is positive at 1 . Thus $\rho<1$ if (and only if)

$$
0<\left(1-\frac{1}{q}\right)\left(1-\frac{r-q}{r}\right)-\frac{s}{2 r}=\frac{q-1}{r}-\frac{s}{2 r}=\frac{q-1-s / 2}{r},
$$

or equivalently $1+s / 2<q$, as claimed. 
Remark If the hypothesis $1+s / 2<q$ does not hold for $\tau$, it will hold for $\tau^{n}$ for some $n>1$.

A trivial consequence of Theorem 2 is that for all $f \in S_{1}$ and $n \geq 0$,

$$
\left\|P_{\tau}^{n} f-\tilde{f}\right\|_{\alpha, \beta} \leq \rho^{n}\|f-\tilde{f}\|_{\alpha, \beta} .
$$

Our main goal, though, is to use Theorem 2 to estimate how close an approximate invariant density $f$ is to $\tilde{f}$ in terms of how close $f$ is to $P_{\tau} f$ (which can be computed without knowing $\tilde{f})$.

Corollary 3 If $1+s / 2<q$, then for $f, \tilde{f} \in S_{1}$ with $P_{\tau} \tilde{f}=\tilde{f}$,

$$
\|f-\tilde{f}\|_{\alpha, \beta} \leq \frac{1}{(1-\rho)}\left\|f-P_{\tau} f\right\|_{\alpha, \beta} .
$$

Proof By the triangle inequality and the above theorem,

$$
\left.\|f-\tilde{f}\|_{\alpha, \beta} \leq\left\|f-P_{\tau} f\right\|_{\alpha, \beta}+\left\|P_{\tau} f-\tilde{f}\right\|_{\alpha, \beta} \leq\left\|f-P_{\tau} f\right\|_{\alpha, \beta}+\rho\|f-\tilde{f}\|_{\alpha, \beta}\right),
$$

from which (6) follows.

Let $\|g\|_{\infty}$ denote the $L^{\infty}$ norm of $g$.

Lemma 4 For all $g \in S_{0}$,

$$
\|g\|_{1} \leq \frac{1}{2} \operatorname{var}(g)
$$

and

$$
\|g\|_{\infty} \leq \operatorname{var}(g)
$$

Proof We ignore the trivial case when $g=0$ almost everywhere. Since $g$ has integral zero, it must take on both positive and negative values, whence (8) follows immediately. Let $A$ and $B$ be the sets on which $g$ is respectively positive and negative; then

$$
\int_{A} g(x) d x=\int_{B}(-g(x)) d x=\frac{1}{2}\|g\|_{1} .
$$

Then since $|A|+|B| \leq 1$,

$$
\operatorname{var}(g) \geq \max _{A}(g)+\max _{B}(-g) \geq \frac{\int_{A} g}{|A|}+\frac{\int_{B}(-g)}{|B|}=\frac{\|g\|_{1}}{2|A|}+\frac{\|g\|_{1}}{2|B|} \geq 2\|g\|_{1},
$$

which is equivalent to (7).

Corollary 5 If $1+s / 2<q$, then for $f, \tilde{f} \in S_{1}$ with $P_{\tau} \tilde{f}=\tilde{f}$,

$$
\|f-\tilde{f}\|_{1} \leq \frac{1}{2(1-\rho)} \operatorname{var}\left(f-P_{\tau} f\right),
$$

and

$$
\|f-\tilde{f}\|_{\infty} \leq \frac{2 \alpha+\beta}{2 \alpha(1-\rho)} \operatorname{var}\left(f-P_{\tau} f\right) .
$$


Proof Since both $f-\tilde{f}$ and $f-P_{\tau} f$ lie in $S_{0}$, by the previous lemma,

$$
(2 \alpha+\beta)\|f-\tilde{f}\|_{1} \leq\|f-\tilde{f}\|_{\alpha, \beta} \leq \frac{1}{1-\rho}\left\|f-P_{\tau} f\right\|_{\alpha, \beta}, \leq \frac{1}{1-\rho}(\alpha+\beta / 2) \operatorname{var}\left(f-P_{\tau} f\right),
$$

from which (9) follows. Likewise

$$
\alpha\|f-\tilde{f}\|_{\infty} \leq\|f-\tilde{f}\|_{\alpha, \beta} \leq \frac{1}{1-\rho}(\alpha+\beta / 2) \operatorname{var}\left(f-P_{\tau} f\right),
$$

which is equivalent to (10).

Proof of Lemma 1 Notice that (3) is equivalent to (2), which we will prove for all $f \in S$. Now

$$
\frac{d}{d x} P_{\tau} f(x)=\sum_{\tau(y)=x} \frac{d y}{d x} \frac{d}{d y} \frac{f(y)}{\left|\tau^{\prime}(y)\right|}=\sum_{\tau(y)=x} \frac{1}{\tau^{\prime}(y)} \frac{\tau^{\prime}(y) f^{\prime}(y)-\tau^{\prime \prime}(y) f(y)}{\tau^{\prime}(y)^{2}} .
$$

Hence

$$
\begin{aligned}
\operatorname{var}\left(P_{\tau} f\right) & =\int_{T}\left|\frac{d}{d x} P_{\tau} f(x)\right| d x \\
& =\int_{T}\left|\sum_{\tau(y)=x} \frac{1}{\tau^{\prime}(y)} \frac{\tau^{\prime}(y) f^{\prime}(y)-\tau^{\prime \prime}(y) f(y)}{\tau^{\prime}(y)^{2}}\right| d x \\
& \leq \int_{T} \sum_{\tau(y)=x} \frac{1}{\tau^{\prime}(y)}\left|\frac{\tau^{\prime}(y) f^{\prime}(y)-\tau^{\prime \prime}(y) f(y)}{\tau^{\prime}(y)^{2}}\right| d x \\
& =\int_{T}\left|\frac{\tau^{\prime}(y) f^{\prime}(y)-\tau^{\prime \prime}(y) f(y)}{\tau^{\prime}(y)^{2}}\right| d y \\
& \leq \int_{T}\left|\frac{1}{\tau^{\prime}(y)} f^{\prime}(y)\right|+\left|\frac{\tau^{\prime \prime}(y)}{\tau^{\prime}(y)^{2}} f(y)\right| d y \\
& \leq \frac{1}{q} \operatorname{var}(f)+s\|f\|_{1}
\end{aligned}
$$

as claimed.

Next we prove (4), which is only valid for $g \in S_{0}$. We ignore the trivial case when $\left\|P_{\tau} g\right\|_{1}=0$. Let $A$ be the set on which $P_{\tau} g>0$ and $B$ be the set on which $P_{\tau} g<0$. Write $[0,1]=\cup_{j} I_{j}$ where the intervals $I_{j}$ are disjoint (except for their endpoints), and $\tau$ is a $C^{2}$ diffeomorphism from the interior of each $I_{j}$ onto $(0,1)$. Let $A_{j}=\tau^{-1}(A) \cap I_{j}$ and $a_{j}=\int_{A_{j}} g(x) d x$; define $B_{j}$ and $b_{j}$ similarly. Then

$$
\sum_{j} a_{j}=\int_{\tau^{-1}(A)} g(x) d x=\int_{A} P_{\tau} g(x) d x=\frac{1}{2}\left\|P_{\tau} g\right\|_{1}
$$

and likewise

$$
\sum_{j}\left(-b_{j}\right)=\frac{1}{2}\left\|P_{\tau} g\right\|_{1}
$$


Also,

$$
\sum_{j}\left|a_{j}\right|+\sum_{j}\left|b_{j}\right| \leq\|g\|_{1}
$$

Notice that the variation of $g$ on $I_{j}$ must be at least the difference in the average values of $g$ on $A_{j}$ and $B_{j}$. Thus

$$
\operatorname{var}(g) \geq \sum_{j}\left(\frac{a_{j}}{\left|A_{j}\right|}-\frac{b_{j}}{\left|B_{j}\right|}\right)
$$

Let $\alpha=|A|$ and $\beta=|B|$. Then $\alpha+\beta \leq 1$, and since $q \leq\left|\tau^{\prime}\right| \leq r$, it follows that $\alpha / r \leq\left|A_{j}\right| \leq \alpha / q$ and $\beta / r \leq\left|B_{j}\right| \leq \beta / q$. Thus

$$
\begin{aligned}
\operatorname{var}(g) & \geq \sum_{j}\left(\frac{\min \left(q a_{j}, r a_{j}\right)}{\alpha}+\frac{\min \left(-q b_{j},-r b_{j}\right)}{\beta}\right) \\
& =\sum_{j}\left(\frac{(r+q) a_{j}-(r-q)\left|a_{j}\right|}{2 \alpha}+\frac{(r+q)\left(-b_{j}\right)-(r-q)\left|b_{j}\right|}{2 \beta}\right) \\
& =\frac{(r+q) \sum_{j} a_{j}-(r-q) \sum_{j}\left|a_{j}\right|}{2 \alpha}+\frac{(r+q) \sum_{j}\left(-b_{j}\right)-(r-q) \sum_{j}\left|b_{j}\right|}{2 \beta} .
\end{aligned}
$$

Case 1: One of the numerators in (11), say the first one, is negative. Then

$$
\|g\|_{1} \geq \sum_{j}\left|a_{j}\right|+\sum_{j}\left|b_{j}\right| \geq \frac{r+q}{r-q} \sum_{j} a_{j}+\sum_{j}\left(-b_{j}\right)=\left(\frac{r+q}{r-q}+1\right) \frac{1}{2}\left\|P_{\tau} g\right\|_{1}=\frac{r}{r-q}\left\|P_{\tau} g\right\|_{1},
$$

whence (3) is proved.

Case 2: Both numerators in (11) are nonnegative. Let $y$ be the first numerator and $z$ the second numerator. Given that $\alpha+\beta \leq 1$, one finds that $y /(2 \alpha)+z /(2 \beta)$ is minimized when $\alpha=\sqrt{y} /(\sqrt{y}+\sqrt{z})$ and $\beta=\sqrt{z} /(\sqrt{y}+\sqrt{z})$, and hence

$$
\frac{y}{2 \alpha}+\frac{z}{2 \beta} \geq \frac{1}{2}(\sqrt{y}+\sqrt{z})^{2}=\frac{y+z}{2}+\sqrt{y z} \geq 2 \min (y, z)=2(y+z-\max (y, z)) .
$$

Now

$$
y \leq(r+q) \sum_{j} a_{j}-(r-q) \sum_{j} a_{j}=2 q \sum_{j} a_{j}=q\left\|P_{\tau} g\right\|_{1},
$$

and likewise $z \leq q\left\|P_{\tau} g\right\|_{1}$. Thus

$$
\begin{aligned}
\operatorname{var}(g) & \geq 2(y+z-\max (y, z)) \\
& \geq 2\left(y+z-q\left\|P_{\tau} g\right\|_{1}\right) \\
& =2\left((r+q)\left(\sum_{j} a_{j}+\sum_{j}\left(-b_{j}\right)\right)-(r-q)\left(\sum_{j}\left|a_{j}\right|+\sum_{j}\left|b_{j}\right|\right)-q\left\|P_{\tau} g\right\|_{1}\right) \\
& \geq 2\left((r+q)\left\|P_{\tau} g\right\|_{1}-(r-q)\|g\|_{1}-q\left\|P_{\tau} g\right\|_{1}\right) \\
& =2 r\left\|P_{\tau} g\right\|_{1}-2(r-q)\|g\|_{1},
\end{aligned}
$$

from which (3) easily follows. 


\section{Rigorous estimates with a computer}

We next explore what we can show in general about bounding the error in a computer estimate of the time average without knowing the specific form of the map $\tau$ or the function $\varphi$ to be averaged. Our goal is to be able to compute rigorous bounds on the integral

$$
\int_{0}^{1} \varphi(x) \tilde{f}(x) d x
$$

where $\tilde{f}$ is the invariant density for $\tau$. To approximate this integral we can compute an approximate invariant density $f$ and numerically integrate $\varphi f$. The total error $E$ is bounded by the sum of the error $E^{\text {num }}$ in the numerical integration procedure and the error

$$
E^{a p p}=\left|\int_{0}^{1} \varphi(x)(f(x)-\tilde{f}(x)) d x\right|
$$

due to the approximation of $\tilde{f}$. By Corollary 5 , it follows that

$$
E^{a p p} \leq\|\varphi\|_{L^{1}}\|f-\tilde{f}\|_{L^{\infty}} \leq \frac{2 \alpha+\beta}{2 \alpha(1-\rho)}\|\varphi\|_{L^{1}} \operatorname{var}\left(f-P_{\tau} f\right) .
$$

We shall describe in general how to estimate $\operatorname{var}\left(f-P_{\tau} f\right)$.

Remark In our eventual application, $\varphi$ is unbounded but integrable, so we assume only that $\varphi \in L^{1}[0,1]$. If $\varphi$ were bounded it would probably be preferable to work in terms of the $L^{\infty}$ norm of $\varphi$ and the $L^{1}$ norm of $f-\tilde{f}$.

Assume that $\tau$ is in fact $C^{3}$ on all its branches, with $\left|\tau^{\prime \prime \prime}\right| \leq K$. Assume that in doing computations we divide $[0,1]$ into $N$ equal intervals and keep track of $f$ at the endpoints of these intervals (henceforth these $N+1$ points will be know as the "grid points"). We think of $f$ as a piecewise linear function which is linear on each of the $N$ intervals and continuous at the grid points; such an $f$ is uniquely determined by its values at the grid points. Assume we can rigorously show that $\left|f-P_{\tau} f\right| \leq \delta$ at each grid point, and assume the following bounds on $f$ :

$$
|f| \leq C_{0}, \quad\left|f^{\prime}\right| \leq C_{1}, \quad\left|f^{\prime}(x)-f^{\prime}(x+1 / N)\right| \leq \frac{C_{2}}{N}
$$

Finally, recall that $1<q \leq\left|\tau^{\prime}\right| \leq r$ and $\left|\tau^{\prime \prime}\right| / \tau^{\prime 2} \leq s$.

Proposition 6 Given the hypotheses in the above paragraph,

$$
\operatorname{var}\left(f-P_{\tau} f\right) \leq \max \left(2 N \delta, \frac{m}{N q}\left(\frac{C_{2}}{q}+\frac{3 s C_{1}}{q}+3 s^{2} C_{0}+\frac{K}{q^{3}} C_{0}\right)\right) .
$$


Proof Since $f$ is continuous, so is $P_{\tau} f$, and $\operatorname{var}\left(f-P_{\tau} f\right)$ is at most $N$ times the maximum variation of $f-P_{\tau} f$ between any two consecutive grid points. Thus

$$
\operatorname{var}\left(f-P_{\tau} f\right) \leq N \max \left(2 \delta, \frac{1}{N} \sup _{|x-\tilde{x}| \leq 1 / N}\left|\left(P_{\tau} f\right)^{\prime}(x)-\left(P_{\tau} f\right)^{\prime}(\tilde{x})\right|\right) .
$$

Let $m$ be the number of branches $\tau$ has. Then

$$
\begin{aligned}
& \sup _{|x-\tilde{x}| \leq \frac{1}{N}}\left|\left(P_{\tau} f\right)^{\prime}(x)-\left(P_{\tau} f\right)^{\prime}(\tilde{x})\right| \\
& \leq m \sup _{|y-\tilde{y}| \leq \frac{1}{N q}}\left|\frac{\tau^{\prime}(y) f^{\prime}(y)-\tau^{\prime \prime}(y) f(y)}{\tau^{\prime}(y)^{3}}-\frac{\tau^{\prime}(\tilde{y}) f^{\prime}(\tilde{y})-\tau^{\prime \prime}(\tilde{y}) f(\tilde{y})}{\tau^{\prime}(\tilde{y})^{3}}\right| \\
& \leq m\left(\sup _{|y-\tilde{y}| \leq \frac{1}{N q}}\left|\frac{f^{\prime}(y)}{\tau^{\prime}(y)^{2}}-\frac{f^{\prime}(\tilde{y})}{\tau^{\prime}(\tilde{y})^{2}}\right|+\sup _{|y-\tilde{y}| \leq \frac{1}{N q}}\left|\frac{\tau^{\prime \prime}(y)}{\tau^{\prime}(y)^{3}} f(y)-\frac{\tau^{\prime \prime}(\tilde{y})}{\tau^{\prime}(\tilde{y})^{3}} f(\tilde{y})\right|\right) \\
& \leq m\left(\frac{1}{q^{2}} \sup _{|y-\tilde{y}| \leq \frac{1}{N q}}\left|f^{\prime}(y)-f^{\prime}(\tilde{y})\right|+C_{1} \sup _{|y-\tilde{y}| \leq \frac{1}{N q}\left|\frac{1}{\tau^{\prime}(y)^{2}}-\frac{1}{\tau^{\prime}(\tilde{y})^{2}}\right|}\right. \\
&\left.+\frac{s}{q} \sup _{|y-\tilde{y}| \leq \frac{1}{N q}}|f(y)-f(\tilde{y})|+C_{0} \sup _{|y-\tilde{y}| \leq \frac{1}{N q}}\left|\frac{\tau^{\prime \prime}(y)}{\tau^{\prime}(y)^{3}}-\frac{\tau^{\prime \prime}(\tilde{y})}{\tau^{\prime}(\tilde{y})^{3}}\right|\right) \\
& \leq m\left(\frac{1}{q^{2}} \frac{C_{2}}{N}+C_{1} \frac{1}{N q} \frac{2 s}{q}+\frac{s}{q} \frac{1}{N q} C_{1}+C_{0} \frac{1}{N q}\left(\frac{K}{q^{3}}+3 s^{2}\right)\right) \\
&= \frac{m}{N q}\left(\frac{C_{2}}{q}+\frac{3 s C_{1}}{q}+3 s^{2} C_{0}+\frac{K}{q^{3}} C_{0}\right) .
\end{aligned}
$$

The previous two inequalities imply (12).

It then follows that

$$
E^{a p p} \leq \frac{2 \alpha+\beta}{2 \alpha(1-\rho)}\|\varphi\|_{L^{1}} \max \left(2 N \delta, \frac{m}{N q}\left(\frac{C_{2}}{q}+\frac{3 s C_{1}}{q}+3 s^{2} C_{0}+\frac{K}{q^{3}} C_{0}\right)\right) .
$$

In the following section we consider an example for which we can explicitly determine the constants on the right side of the above equation, and bound the remaining error term $E^{\text {num }}$ as well.

\section{The Ruelle quadratic map}

We consider now the quadratic map at the parameter value for which the critical point maps to a fixed point after three iterates. Ruelle [15] proved that this map has an absolutely continuous invariant measure. The argument involves a change of coordinates after which the second iterate of the map is uniformly expanding. Here we obtain a rigorous approximation to the invariant density in the new coordinate system, and in turn rigorously approximate 
some time averages for the map. For brevity, many details of the computation are omitted; some steps are explained more in depth in an earlier version of this paper, available from the author.

\subsection{Convergence of the Frobenius-Perron operator}

Let $\sigma(t)=t^{2}-c$, and let $t_{0}=0$ and $t_{n+1}=\sigma\left(t_{n}\right)$ for $n \geq 0$. We assume $c$ is such that $t_{3}$ (the third iterate of the critical point) is a fixed point of $\sigma$ but $t_{2} \neq t_{3}$. The absolutely continuous invariant measure [15] for $\sigma$ is supported on the intervals $\left[t_{1}, t_{3}\right]$ and $\left[t_{3}, t_{2}\right]$, which are exchanged by $\sigma$. In order to estimate this measure, we look at the second iterate of $\sigma$ on the interval $\left[t_{3}, t_{2}\right]$, and make a change of variables on this interval.

Before making the change of variables, let us determine the parameter value at which the above situation occurs. Given that $t_{3}$ is a fixed point but $t_{2}$ is not, it follows (since $\sigma\left(t_{3}\right)=\sigma\left(t_{2}\right)$ but $\left.t_{3} \neq t_{2}\right)$ that $t_{3}=-t_{2}$. Now $t_{2}=c^{2}-c$ and $t_{3}=c^{4}-2 c^{3}+c^{2}-c$, so setting $t_{3}+t_{2}=0$ and throwing out the spurious solution $c=0$ we get

$$
c^{3}-2 c^{2}+2 c-2=0 .
$$

This equation has one real solution $c \approx 1.5437$.

Next let

$$
t=\psi(\theta)=\left(c-c^{2}\right) \cos (\pi \theta), \quad \theta \in[0,1],
$$

and let

$$
\tau(\theta)=\psi^{-1}(\sigma(\sigma(\psi(\theta))))
$$

Now

$$
\sigma(\sigma(t))=\left(t^{2}-c\right)^{2}-c=t^{4}-2 c t^{2}+c^{2}-c,
$$

and since $t_{3}=c-c^{2}$ is a fixed point of $\sigma$, it follows that

$$
\left(c-c^{2}\right)^{4}=2 c\left(c-c^{2}\right)^{2}+2\left(c-c^{2}\right) .
$$

Thus

$$
\begin{aligned}
\tau(\theta) & =\frac{1}{\pi} \arccos \left(\frac{\left(c-c^{2}\right)^{4} \cos ^{4}(\pi \theta)-2 c\left(c-c^{2}\right)^{2} \cos ^{2}(\pi \theta)+c^{2}-c}{c-c^{2}}\right) \\
& =\frac{1}{\pi} \arccos \left(\left(2 c\left(c-c^{2}\right)+2\right) \cos ^{4}(\pi \theta)-2 c\left(c-c^{2}\right) \cos ^{2}(\pi \theta)-1\right) \\
& =\frac{1}{\pi} \arccos \left(\frac{1}{2}\left(c\left(c-c^{2}\right)+1\right)(\cos (2 \pi \theta)+1)^{2}-c\left(c-c^{2}\right)(\cos (2 \pi \theta)+1)-1\right) \\
& =\frac{1}{\pi} \arccos \left(\frac{1}{2}\left(c\left(c-c^{2}\right)+1\right) \cos ^{2}(2 \pi \theta)+\cos (2 \pi \theta)-\frac{1}{2}\left(c\left(c-c^{2}\right)+1\right)\right)
\end{aligned}
$$


that is,

$$
\tau(\theta)=\frac{1}{\pi} \arccos \left(\cos (2 \pi \theta)+\kappa \sin ^{2}(2 \pi \theta)\right)
$$

where

$$
\kappa=\frac{c\left(c^{2}-c\right)-1}{2} \approx 0.1478
$$

It then follows that

$$
\begin{aligned}
\tau^{\prime}(\theta) & =-\frac{1}{\pi \sqrt{1-\left(\cos (2 \pi \theta)+\kappa \sin ^{2}(2 \pi \theta)\right)^{2}}}(-2 \pi \sin (2 \pi \theta)+4 \pi \kappa \sin (2 \pi \theta) \cos (2 \pi \theta)) \\
& =\frac{2 \sin (2 \pi \theta)(1-2 \kappa \cos (2 \pi \theta))}{\sqrt{\sin ^{2}(2 \pi \theta)\left(1-2 \kappa \cos (2 \pi \theta)-\kappa^{2} \sin ^{2}(2 \pi \theta)\right)}}
\end{aligned}
$$

that is,

$$
\tau^{\prime}(\theta)= \pm \frac{2(1-2 \kappa \cos (2 \pi \theta))}{\sqrt{(1-\kappa \cos (2 \pi \theta))^{2}-\kappa^{2}}}
$$

where the plus sign is valid for $\theta<1 / 2$ and the minus sign for $\theta>1 / 2$. One then finds that

$$
\tau^{\prime \prime}(\theta)=4 \pi \kappa|\sin (2 \pi \theta)| \frac{1-2 \kappa^{2}-\kappa \cos (2 \pi \theta)}{\left((1-\kappa \cos (2 \pi \theta))^{2}-\kappa^{2}\right)^{\frac{3}{2}}} .
$$

In particular, $\tau^{\prime \prime}$ is positive, so $\tau^{\prime}(0) \leq\left|\tau^{\prime}\right| \leq\left|\tau^{\prime}(1 / 2)\right|$, and we find that $q<\left|\tau^{\prime}\right|<r$ with

$$
q=1.678, \quad r=2.277
$$

Next we compute $\tau^{\prime \prime \prime}(\theta)$, and it is not hard to show that $\left|\tau^{\prime \prime \prime}(\theta)\right|<20$. This allows us to rigorously bound $\left|\tau^{\prime \prime}\right| / \tau^{\prime 2}$ by computing the latter function at a large number (specifically, $\left.10^{8}\right)$ evenly spaced points in $[0,1]$. We find $\left|\tau^{\prime \prime}\right| / \tau^{\prime 2}<s$ with

$$
s=0.535 \text {. }
$$

Recall that the matrix $M$ from which the constants $\alpha, \beta, \rho$ in Corollary 5 are computed is

$$
M=\left(\begin{array}{cc}
\frac{1}{q} & s \\
\frac{1}{2 r} & \frac{r-q}{r}
\end{array}\right) .
$$

The largest eigenvalue $\rho$ of $M$ is found to satisfy $\rho<0.811$, while the coordinates $(\alpha, \beta)$ of the left eigenvector corresponding to $\rho$ satisfy $\beta / \alpha<0.978$. Thus

$$
\frac{2 \alpha+\beta}{2 \alpha(1-\rho)}=\frac{1+\frac{\beta}{2 \alpha}}{1-\rho}<\frac{1.489}{0.189}<7.88 \text {. }
$$




\subsection{The invariant density}

To find an approximate invariant density for $\tau$, the idea is to iterate the Frobenius-Perron operator $P_{\tau}$ starting with a uniform invariant density. Notice that $\tau$ maps $[0,1 / 2]$ onto $[0,1]$ and that $\tau(1-\theta)=\tau(\theta)$. Let $\tau^{-1}(\theta)$ be the branch of the inverse of $\tau$ with values in $[0,1 / 2]$. Then

$$
P_{\tau} f(\theta)=\frac{f\left(\tau^{-1}(\theta)\right)}{\left|\tau^{\prime}\left(\tau^{-1}(\theta)\right)\right|}+\frac{f\left(1-\tau^{-1}(\theta)\right)}{\left|\tau^{\prime}\left(1-\tau^{-1}(\theta)\right)\right|} .
$$

From (14) we find that

$$
\tau^{-1}(\theta)=\frac{1}{2 \pi} \arccos \left(\frac{1-\sqrt{1+4 \lambda^{2}-4 \lambda \cos (\pi \theta)}}{2 \lambda}\right) .
$$

Using this and (15) we can program a computer with the precise formula for $P_{\tau}$.

Recall that on the computer we keep track of densities at the $N+1$ "grid points" $0,1 / N, 2 / N, \ldots, 1$ and regard the densities to be linear between the grid points. Thus we interpolate to find the values of $f\left(\tau^{-1}(\theta)\right)$ and $f\left(1-\tau^{-1}(\theta)\right)$ in evaluating $P_{\tau} f$ at a given grid point $\theta$. One finds that the computer approximation to $P_{\tau} f$ does not have integral one, thus we normalize the density after each computer iterate of $P_{\tau}$. We use $N=10^{5}$ and iterate (starting with a uniform density) until the unnormalized computer approximation to $P_{\tau} f$ is within $10^{-13}$ of $f$.

Henceforth $f$ will represent the computer approximation (found as described above) to the invariant density $\tilde{f}$ of $P_{\tau}$. In order to estimate the error $\|f-\tilde{f}\|_{L^{\infty}}$ we must first estimate how close $P_{\tau} f$ (not its computer approximation) is to $f$ at the grid points. The error in the computer approximation to $P_{\tau} f$ is due mainly to errors in computing the inverse cosine; a careful analysis of the error yields the bound $\left|f-P_{\tau} f\right|<10^{-9}$ for every grid point. We thus let $\delta=10^{-9}$.

Since the values of $f$ at the grid points are known and $f$ is linear in between, it is trivial to find values of $C_{0}, C_{1}$, and $C_{2}$ which satisfy the hypotheses of Proposition 6 . We find that

$$
C_{0}=1.245, \quad C_{1}=0.737, \quad C_{2}=5.961
$$

suffice. Recall that we found values for $q, s$, and $K=20$ in the previous section. Then by Proposition 6,

$$
\operatorname{var}\left(f-P_{\tau} f\right) \leq \max \left(2 \cdot 10^{-4}, 1.27 \cdot 10^{-4}\right)=2 \cdot 10^{-4} .
$$

Thus for any $L^{1}$ function $\varphi$,

$$
E^{a p p}=\left|\int_{0}^{1} \varphi(\theta) f(\theta) d \theta-\int_{0}^{1} \varphi(\theta) \tilde{f}(\theta) d \theta\right| \leq 7.88 \cdot 2 \cdot 10^{-4}\|\varphi\|_{L^{1}}<1.58 \cdot 10^{-3}\|\varphi\|_{L^{1}}
$$

Then for a given $\varphi$ we can rigorously approximate its time average $\int_{0}^{1} \varphi(\theta) \tilde{f}(\theta) d \theta$ by numerically integrating $\varphi f$, bounding the error $E^{\text {num }}$ in that procedure, and bounding $\|\varphi\|_{L^{1}}$ in order to bound the total error. 


\subsection{Lyapunov exponents}

We are now in a position to estimate the Lyapunov exponents of the complex map $z \mapsto$ $z^{2}-(1+\lambda i) \bar{z}$, or equivalently the planar map

$$
\begin{gathered}
x \mapsto x^{2}-y^{2}-x-\lambda y, \\
y \mapsto 2 x y-\lambda x+y,
\end{gathered}
$$

where $\lambda$ is a positive constant to be specified. The line $y=\lambda / 2$ is invariant under this map, and on this line the map acts according to

$$
x \mapsto x^{2}-x-\frac{3 \lambda^{2}}{4} .
$$

For a value of $\lambda$ to be determined shortly, this one-dimensional map has a chaotic attractor $A$. It follows that $A$ is an invariant set for the two-dimensional map, and our goal is to estimate the Lyapunov exponents of this map on $A$. One exponent will simply be the Lyapunov exponent of the one-dimensional map, and the other will reflect the rate of contraction or expansion transverse to $A$ for the two-dimensional map.

This planar map, originally studied in [12], has been of great interest recently as a fundamental example of the phenomenon of "riddled" and "intermingled" basins of attraction $[2,1]$. In order to verify mathematically the properties of this map which were discovered with a computer, it is necessary to verify that the "transverse" Lyapunov exponent for $A$ is negative, which in particular implies that $A$ attracts a set of positive Lebesgue dimensional measure.

Let $t=x-1 / 2$ and $c=3\left(\lambda^{2}+1\right) / 4$; then the map on $A$ can be written

$$
t \mapsto t^{2}-c
$$

which is of course the map studied in Section 4.1. The equation for $\lambda$ corresponding to (13) is

$$
27 \lambda^{6}+9 \lambda^{4}+33 \lambda^{2}-77=0
$$

which has one positive solution $\lambda \approx 1.0287$. Thus for this value of $\lambda$, the invariant set $A$ has an absolutely continuous invariant measure $\mu$ which we approximated in Section 4.2. (More precisely, we approximated the invariant density for the second iterate of the map in a different coordinate system.)

By the multiplicative ergodic theorem [14], almost every point on $A$ has the same Lyapunov exponents, and since the Jacobian matrix of the two-dimensional map on the invariant line $y=\lambda / 2$ is

$$
\left(\begin{array}{cc}
2 x-1 & -2 \lambda \\
0 & 2 x+1
\end{array}\right)
$$


it can be shown that the Lyapunov exponents of this map on $A$ are the averages of the functions $\log |2 x-1|$ and $\log |2 x+1|$ with respect to $\mu$. Since $x=t+1 / 2=\left(c-c^{2}\right) \cos (\pi \theta)+$ $1 / 2$ and the invariant density $\tilde{f}$ we estimated in Section 4.2 is for the second iterate of our map, the Lyapunov exponents are the integrals of the following functions multiplied by $\tilde{f}$ :

$$
\begin{aligned}
\varphi_{1}(\theta) & =\frac{1}{2}\left(\log \left|2\left(c-c^{2}\right) \cos (\pi \theta)\right|+\log \left|2\left(\left(c-c^{2}\right)^{2} \cos ^{2}(\pi \theta)-c\right)\right|\right) \\
& =\frac{1}{2} \log \left|4 c^{2}(c-1) \cos (\pi \theta)\left(1-c(c-1)^{2} \cos ^{2}(\pi \theta)\right)\right| . \\
\varphi_{2}(\theta)= & \frac{1}{2}\left(\log \left|2\left(c-c^{2}\right) \cos (\pi \theta)+2\right|+\log \left|2\left(\left(c-c^{2}\right)^{2} \cos ^{2}(\pi \theta)-c\right)+2\right|\right) \\
= & \frac{1}{2} \log \left|4(c-1)(1-c(c-1) \cos (\pi \theta))\left(1-c^{2}(c-1) \cos ^{2}(\pi \theta)\right)\right| .
\end{aligned}
$$

To estimate the Lyapunov exponents, we numerically integrate $\varphi_{1} f$ and $\varphi_{2} f$, where $f$ is the approximate invariant density described in Section 4.2. Because $\varphi_{1}$ and $\varphi_{2}$ have singularities, we must do the numerical integration carefully in order to be able to bound the error in each case. Specifically, we write $\varphi_{1}$ as $(1 / 2) \log |\theta-1 / 2|$ plus a remainder term which is nonsingular; the integral of the logarithm times the piecewise linear function $f$ can be computed exactly in terms of the values of $f$ at the grid points, while the nonsingular term is integrated numerically with a standard error estimate. We treat $\varphi_{2}$ similarly, and bound the total error by $3 \cdot 10^{-8}$ in each case.

Carrying out the computations described in the above paragraph and rounding to 4 places yields

$$
\int_{0}^{1} \varphi_{1}(\theta) f(\theta) d \theta=0.3422+E_{1}^{\text {num }}
$$

where

$$
E_{1}^{\text {num }}<10^{-4} .
$$

Similarly, we find that

$$
\int_{0}^{1} \varphi_{2}(\theta) f(\theta) d \theta=-0.3704+E_{2}^{\text {num }}
$$

where

$$
E_{2}^{\text {num }}<10^{-4} .
$$

In order to bound the remaining error terms $E_{1}^{a p p}$ and $E_{2}^{a p p}$ (due to the difference between $f$ and $\tilde{f}$ ), we need only bound $\left\|\varphi_{1}\right\|_{L^{1}}$ and $\left\|\varphi_{2}\right\|_{L^{1}}$. Again, this involves integrating functions with logarithmic singularities, but doing the numerical integration carefully as described above we find that

$$
\left\|\varphi_{1}\right\|_{L^{1}}<0.460
$$

and

$$
\left\|\varphi_{2}\right\|_{L^{1}}<0.564
$$


. It follows from Section 4.2 that

$$
E_{1}^{a p p}<1.58 \cdot 10^{-3}\left\|\varphi_{1}\right\|_{L^{1}}<7.27 \cdot 10^{-4}
$$

and

$$
E_{2}^{a p p}<1.58 \cdot 10^{-3}\left\|\varphi_{2}\right\|_{L^{1}}<8.92 \cdot 10^{-4} .
$$

Therefore the total error in our approximations to each Lyapunov exponent can be bounded by $10^{-3}$, and we conclude that

$$
0.341<\int_{0}^{1} \varphi_{1}(\theta) f(\theta) d \theta<0.344
$$

and

$$
-0.372<\int_{0}^{1} \varphi_{2}(\theta) f(\theta) d \theta<-0.369 .
$$

In particular, the Lyapunov exponent for the one-dimensional map is positive, and the transverse Lyapunov exponent for the corresponding planar map is negative.

We remark that the computations and error estimates in this example were obtained with standard "double precision" (64-bit) floating point arithmetic; increased precision would of course lead to much sharper error bounds. In light of recent developments concerning the accuracy of floating point arithmetic on certain processors, we should also point out the computations reported here have been double-checked using several different computers, operating systems, and compilers.

\subsection{Perturbations of the Parameter}

The application of the method described in the paper to the example in this section depends on the particular value $c$ of the parameter in the quadratic map. Indeed since there is a stable periodic orbit for a dense set of parameters [19], we cannot even hope that our results extend to an entire neighborhood of $c$. However, by the work of Rychlik and Sorets [18], there is a positive measure set $S$ of parameter values near $c$ for which the quadratic map has an absolutely continuous invariant measure with density function $L^{p}$ close to the invariant density for $c$, for any $p \in[1,2)$. In particular, for parameter values in $S$ the time average of any function in $L^{q}$ for $q>2$ converges to the time average for $c$ as the parameter approaches $c$ (staying within $S$ ). Thus for example our conclusion that the planar map has a negative transverse Lyapunov exponent is valid for a set of parameters with positive Lebesgue measure.

\section{Acknowledgments}

The author would like to thank J. Alexander, I. Kan, M. Rychlik, Ya. Sinai, M. Yakobson, J. Yorke, and L-S. Young for their very helpful comments. 


\section{References}

[1] J. C. Alexander, B. R. Hunt, I. Kan, \& J. A. Yorke, Intermingled basins for the triangle map, Erg. Th. Dyn. Sys. (to appear).

[2] J. C. Alexander, I. Kan, J. A. Yorke, \& Z-P. You, Riddled Basins, Int. J. Bif. Chaos 2 (1992), 795-813.

[3] G. D. Birkhoff, Proof of the ergodic theorem, Proc. Nat. Acad. Sci. 17 (1931), 656-660.

[4] M. L. Blank, A bound for the rate of decrease of correlation in one-dimensional dynamics systems, Functional Anal. Appl. 18 (1984), 50-52.

[5] C. Chiu, Q. Du, \& T. Y. Li, Error estimates of the Markov finite approximation of the Frobenius-Perron operator, Nonlin. Anal. Th. Meth. Appl. 19 (1992), 291-308.

[6] J-P. Eckmann \& D. Ruelle, Ergodic theory of chaos and strange attractors, Rev. Mod. Phys. 57 (1985), 617-656.

[7] F. Hofbauer \& G. Keller, Ergodic properties of invariant measures for piecewise monotonic transformations, Math. Z. 180 (1982), 119-140.

[8] F. Y. Hunt, A Monte Carlo approach to the approximation of invariant measures, National Institute of Standards and Technology Internal Report 4980 (1993).

[9] G. Keller, On the rate of convergence to equilibrium in one-dimensional systems, Comm. Math. Phys. 96 (1984), 181-193.

[10] A. Lasota \& J. A. Yorke, On the existence of invariant measures for piecewise monotonic transformations, Trans. Amer. Math. Soc. 186 (1973), 481-488.

[11] T. Y. Li, Finite approximation for the Frobenius-Perron operator, a solution to Ulam's conjecture, J. Approx. Theory 17 (1976), 177-186.

[12] A. Lopes, Dynamics of real polynomials on the plane and triple point phase transition, Math. Comp. Mod. 13 (1990), 17-32.

[13] W. M. Miller, Stability and approximation of invariant measures for a class of nonexpanding transformations, Nonlin. Anal. Th. Meth. Appl. 23 (1994), 1013-1025.

[14] V. I. Oseledec, A multiplicative ergodic theorem: Liapunov characteristic numbers for dynamical systems, Trans. Moscow Math. Soc. 19 (1968), 197-231. 
[15] D. Ruelle, Applications conservant une mesure absolument continue par rapport à $d x$ sur $[0,1]$, Comm. Math. Phys. 55 (1977), 47-51.

[16] M. Rychlik, Bounded variation and invariant measures, Studia Math. 76 (1983), 69-80.

[17] M. Rychlik, Regularity of the metric entropy for expanding maps, Trans. Amer. Math. Soc. 315 (1989), 833-847.

[18] M. Rychlik \& E. Sorets, Regularity and other properties of absolutely continuous invariant measures for the quadratic family, Comm. Math. Phys. 150 (1992), 217-236.

[19] G. Świątek, Hyperbolicity is dense in the real quadratic family, SUNY Stony Brook IMS preprint \#1992/10, revised 1994.

[20] Richard S. Varga, Matrix Iterative Analysis, Prentice-Hall, Englewood Cliffs, NJ, 1962.

[21] L-S. Young, Decay of correlations for certain quadratic maps, Comm. Math. Phys. 146 (1992), 123-138. 